\title{
BMJ Open Association between fasting blood glucose levels and stroke events: a large- scale community-based cohort study from China
}

\author{
Ya Zhang (D) , ${ }^{1,2}$ Shujun Gu, ${ }^{3}$ Cuicui Wang, ${ }^{1,2}$ Dong Liu, ${ }^{1,2}$ Qiuyi Zhang, ${ }^{3}$ Man Yang, ${ }^{3}$ \\ Zhengyuan Zhou, ${ }^{3}$ Hui Zuo ${ }^{1,2}$
}

To cite: Zhang Y, Gu S, Wang C, et al. Association between fasting blood glucose levels and stroke events: a largescale community-based cohort study from China. BMJ Open 2021;11:e050234. doi:10.1136/ bmjopen-2021-050234

- Prepublication history and additional supplemental material for this paper are available online. To view these files, please visit the journal online. (http://dx.doi.org/10.1136/ bmjopen-2021-050234).

YZ and SG contributed equally.

Received 15 February 2021 Accepted 03 August 2021

Check for updates

(C) Author(s) (or their employer(s)) 2021. Re-use permitted under CC BY-NC. No commercial re-use. See rights and permissions. Published by BMJ.

${ }^{1}$ School of Public Health, Soochow University Medical College, Suzhou, China

${ }^{2}$ Jiangsu Key Laboratory of Preventive and Translational Medicine for Geriatric Diseases, Medical College of Soochow University, Suzhou, China

${ }^{3}$ Changshu Center for Disease Control and Prevention, Suzhou, China

Correspondence to

Dr Hui Zuo; zuohui@suda.edu.cn

\section{ABSTRACT}

Objectives Diabetes mellitus has been associated with stroke. However, the association between fasting blood glucose (FBG) and stroke risk in a general population remains not clear. The purpose of our study was to examine the FBG levels on subsequent stroke risk in a community-based cohort in China.

Design Prospective cohort study, employing Cox proportional hazard model to analyse the association of FBG levels with stroke risk.

Setting A community-based cohort study included adults participating in a baseline survey conducted in 2013 in Changshu, eastern China.

Participants 16113 participants were recruited with a multistage sampling method, excluding participants with severe disability, severe cancer, severe psychiatric disturbance or previous stroke before enrolment.

Primary outcome measures Stroke events.

Results During a median follow-up of 5.5 years, 417 incident cases of stroke were identified. The adjusted HR for total and ischaemic stroke for participants in the fourth quartile of FBG compared with the first quartile was 1.44 (95\% Cl 1.07 to 1.94$)$ and 1.57 (95\% $\mathrm{Cl} 1.11$ to 2.21$)$, respectively. FBG levels of $\geq 7.0 \mathrm{mmol} / \mathrm{L}$ were associated with an increased risk of stroke based on two clinical classifications (American Diabetes Association: 1.68 (1.24 to 2.27); WHO: $1.62(1.21,2.13))$. In stratified analyses, risk associations existed in women (HR: $1.92,95 \% \mathrm{Cl}$ 1.22 to 3.01 ) and postmenopausal women (HR: 1.68, $95 \% \mathrm{Cl} 1.06$ to 2.68 ) for the fourth quartile versus the first. More importantly, the meta-analysis observed a positive association between FBG levels and stroke risk (pooled HR: $1.70,95 \% \mathrm{Cl} 1.27$ to $2.29 ; n=7))$.

Conclusions Higher FBG level was independently associated with an increased risk of stroke in Chinese adults, especially significant in women.

\section{INTRODUCTION}

Stroke is one of the most important causes of human diseases and death. ${ }^{1}$ According to the statistics of global disease burden in 2013, about 25.7 million patients with stroke and 6.5 million stroke deaths worldwide. ${ }^{2}$ There are 113 million disability-adjusted life years caused by stroke each year. ${ }^{3}$ It poses a
Strengths and limitations of this study

Large sample size, measurement of plasma fasting blood glucose and complete follow-up.

- The meta-analysis based on six previously published studies and the current study further confirmed the association between fasting blood glucose levels and stroke risk.

- No data available on haemoglobin A1c measurements in our study.

heavy psychological and economic burden to patients' families and society. Therefore, the primary prevention of stroke has now become a top priority for global public health. In China, it has become the leading cause of death $^{4}$ and one of the important reasons for disability in adults. ${ }^{5}$

Studies have shown that diabetes mellitus can increase the risk of stroke. ${ }^{67}$ A cohort study involving 510000 people observed that diabetes significantly increased the risk of ischaemic stroke and haematencephalon. ${ }^{7} \mathrm{~A}$ prospective study showed that insulin resistance or diagnosed diabetes can predict the first stroke. ${ }^{8}$ However, these studies focus on diabetic status instead of continuous glucose levels as the study exposure, which limits the generalisability of the research findings. Since fasting blood glucose (FBG) is the least interfered by diet, ${ }^{9}$ the FBG levels are considered as a more reliable tool to measure blood glucose levels than random blood glucose levels. ${ }^{10} \mathrm{FBG}$ levels have a stronger predictive power for functional prognosis than random blood glucose levels. Therefore, rather than focusing on random blood glucose, it is important to explore the association between FBG levels and the risk of stroke.

In this study, a community population from China was followed up for 5.5 years to study the relationship between FBG levels and the 
incidence of stroke, aiming at providing suggestions and a basis for primary prevention of stroke among people with different FBG levels.

\section{METHODS}

\section{Study population}

The baseline survey of the current cohort study was conducted in 2013. The source population was 20343 participants aged 35-74 years in rural communities in Changshu, eastern China who participated in an earlier study on metabolic syndrome in 2008, in addition to a small proportion of new participants. A multistage sampling method was used to recruit participants. Individuals with severe disability, severe cancer or severe psychiatric disturbance were excluded. Of these, 16457 subjects in total were included in the baseline survey. We excluded 137 patients with a previous stroke before enrolment. Participants with missing data on baseline health information $(\mathrm{n}=207)$ were also excluded, leaving 16113 participants in total for final analyses (online supplemental appendix 1).

\section{Laboratory measurements}

Blood samples were collected in the morning after overnight fasting and were evaluated biochemical and clinical parameters on the same day. FBG was measured by an oxidase enzymatic method. The levels of high-density lipoprotein cholesterol (HDL-C), total cholesterol (TC) and triglyceride (TG) were assessed enzymatically using commercial reagents with an automatic biochemistry analyser (Hitachi, Tokyo, Japan). The levels of low-density lipoprotein cholesterol (LDL-C) were calculated by the Friedewald formula. ${ }^{11}$ The estimated glomerular filtration rate (eGFR) was calculated on the basis of the chronic kidney disease-epidemiology creatinine equation ${ }^{12}$.

\section{Follow-up and ascertainment of stroke}

Follow-up began at the baseline and ended at the date of stroke diagnosis, death or, 31 December 2018, whichever came first. We classified total stroke into an ischaemic stroke and non-ischaemic stroke (including haemorrhagic stroke, subarachnoid haemorrhage and unexplained types of stroke). The primary outcome was hospitalisation or death due to stroke collected by ICD-10 codes (total stroke: I60-I61, I63-I64; ischaemic stroke: I63 except I63.9). If a participant had more than one stroke event during the follow-up, only the first stroke was considered.

\section{Assessments of covariates}

Demographic characteristics, lifestyle factors, personal medical history (cardiovascular disease (CVD), hyperlipidaemia, hypertension, diabetes, chronic obstructive pulmonary disease (COPD), nephropathy and cancer) and family history data for all participants were obtained using standard questionnaires administered by trained staff. After the subjects rested for $5 \mathrm{~min}$, the trained observers used an electronic sphygmomanometer (Omron Hp1300, OMRON Corporation, China) to measure the blood pressure in the sitting position three times every $30 \mathrm{~s}$. The criteria for defining hypertension were an average measurement of systolic blood pressure $\geq 140 \mathrm{mmHg}$ or diastolic blood pressure $\geq 90$ mmHg, ${ }^{13}$ diagnosis of hypertension or antihypertensive medication. Impaired fasting glucose (IFG) is defined as an FBG of $6.1-6.9 \mathrm{mmol} / \mathrm{L}$ by $\mathrm{WHO}^{14}$ and an FBG of $5.6-6.9 \mathrm{mmol} / \mathrm{L}$ by the American Diabetes Association (ADA) ${ }^{15}$ The body mass index (BMI) was calculated as weight in kilograms divided by the square of height in metres, and weight and height were measured by standard methods. ${ }^{16}$

\section{Meta-analysis}

We conducted a meta-analysis combining the current study findings with previous observations on FBG and stroke risk. We searched PubMed, Web of Knowledge, medRxiv and bioRxiv databases for related research articles published between 1 January 2000 and 10 September 2020 using the keywords 'stroke', 'cardiovascular disease' and 'fasting blood glucose', 'blood glucose', 'fasting glucose', 'fasting plasma glucose', 'glucose', in addition to 'prospective study', 'cohort study' or 'follow-up'. The study subjects were restricted to apparently healthy adults in the community. Two authors extracted the data independently, and if they had disagreements, discussed it with the third author. This study was conducted following the Preferred Reporting Items for Systematic Reviews and Meta-Analyses guideline. Finally, seven studies were included. ${ }^{17-22}$ We extracted information about authors, sample size, the country where the study was implemented, time of follow-up, adjusted covariates, outcome (stroke events) and the effect size (HR). The measurement and definition of FBG levels were directly extracted from each literature. We used a random-effect model to calculate the pooled risk estimation based on the assumption of study heterogeneity. The Begg and Egger tests were performed to detect potential publication bias. ${ }^{23}$

\section{Statistical analysis}

Baseline demographic characteristics for continuous variables were expressed as median (IQRs), and categorical variables were presented as percentages. Logtransformation was used to all laboratory measures to normalise distributions.

Cox proportional hazard analyses were conducted to evaluate FBG levels and risk of stroke and results presented as HR and 95\% CIs. FBG levels were included in the models as a categorical measure (quartiles and three categories by clinical criteria). Multivariable models included conventional risk factors at baseline: age (continuous), sex, BMI (continuous), current smoking (yes/no), family history of stroke (yes/no), hypertension (yes/no), eGFR $\left(\mathrm{mL} / \mathrm{min} / 1.73 \mathrm{~m}^{2}\right)$ LDL-C $(\mathrm{mmol} / \mathrm{L})$, HDL-C $(\mathrm{mmol} / \mathrm{L})$, TC $(\mathrm{mmol} / \mathrm{L})$, TG $(\mathrm{mmol} / \mathrm{L})$ and use of statins. We also used clinical classification (normal 
FBG, IFG, hyperglycaemic) to classify FBG levels. In addition, we conducted stratified analyses by sex to check potential effect modification. Sensitivity analyses were conducted to determine the robustness of the primary analysis results. We limited the risk association analyses to participants who did not report their own medical history, including hypertension, hyperlipidaemia, cancer, nephropathy, CVD or COPD at baseline.

Statistical analyses were conducted with R (V.4.0.2, www. r-project.org). All tests were two sided, and a p value $<0.05$ was considered statistically significant.

\section{Patient and public involvement}

Patients and the public were not involved in the design, conduct or reporting of this study.

\section{RESULTS}

\section{Characteristics of the study population}

After a median follow-up of 5.5 years (range: 0.1-5.7 years), a total of 417 (192 men and 225 women) stroke events were identified, of which $323(77.5 \%)$ were ischaemic stroke. Table 1 shows the baseline characteristics of participants across the quartiles of FBG. Compared with the participants in the study with lower FBG, those with higher FBG levels were more likely to suffer from obesity, hypertension and hyperlipidaemia and had lower levels of eGFR and preferred to have a family history of stroke $(\mathrm{p}<0.001$, table 1$)$.

\section{FBG levels and risk of stroke}

As shown in table 2, we observed that higher FBG levels were significantly correlated with an increased risk of total and ischaemic stroke in both the original models and models adjusted for age, sex, BMI, HDL-C, LDL-C, TC, TG, eGFR, hypertension, smoking status, use of statins and family history of stroke. Compared with the lowest FBG quartile, the multivariable-adjusted HRs for the highest quartile were 1.44 (95\% CI 1.07 to 1.94 , $\mathrm{p}=0.021)$ for total and 1.57 (95\% CI 1.11 to $2.21, \mathrm{p}=0.016)$ for ischaemic stroke. A positive association between FBG levels and non-ischaemic stroke was observed in the crude model, but the risk association was rendered statistically non-significant in adjusted models. Furthermore, we examined the association between clinical classifications of FBG levels and stroke risk, and the results are presented in table 3. The multivariable-adjusted HR for total stroke was 1.68 (95\% CI 1.24 to $2.27, \mathrm{p}=0.001)$ and $1.62(95 \%$ CI 1.21 to $2.13, \mathrm{p}=0.002)$ for participants with hyperglycaemic compared with those with normal blood glucose, following the $\mathrm{ADA}$ and $\mathrm{WHO}$ recommendations, respectively.

Results of sex-stratified analyses are shown in table 4. In original models, higher levels of FBG were significantly associated with an increased risk of total stroke only in women. After adjustment covariate for age, BMI and other variates, the risk association remained significant (HR: 1.92, 95\% CI 1.22 to 3.01, p=0.004) for the fourth level in female groups. Similar risk estimates were found in postmenopausal or oophorectomy women. But no significant association with stroke was observed for FBG levels in premenopausal women (online supplemental appendix 2).

In sensitivity analyses, the study population was restricted to 15404 subjects without a self-reported history of hyperlipidaemia, CVD, cancer, nephropathy and COPD yielded essentially no change in risk of total stroke (HR: $1.38,95 \%$ CI 1.01 to 1.92 ) and ischaemic stroke (HR: 1.55, 95\% CI 1.06 to 2.26). After further elimination of participants with hypertension, the association in women was slightly raised for total stroke (HR: 2.50, 95\% CI 1.11 to 5.60) and ischaemic stroke (HR: 2.59, 95\% CI 1.03 to 6.51 ) (data not shown).

\section{Meta-analysis}

Figure 1 shows the results of meta-analysis including six previously published prospective studies together with our current study. In a word, higher FBG levels were associated with an increased risk of stroke (pooled HR: 1.70, 95\% CI 1.27 to 2.29). The Begg and Egger tests did not suggest the existence of potential publication bias $(p>0.1$ for both tests).

\section{DISCUSSION}

\section{Principal findings}

In our prospective community-based cohort, higher FBG levels were associated with an increased risk of total and ischaemic stroke. The risk association was prominent in women. After excluding the baseline of hypertension, hyperlipidaemia, CVD, cancer, nephropathy and COPD, these associations were still persisted in analyses of women. Our findings extend the previous knowledge on diabetes and stroke risk, suggesting an important role of routine glucose measurement in stroke prevention.

\section{Associations of FBG with stroke risk}

When analysing stroke subtypes, there is no evidence that FBG is associated with non-ischaemic stroke, but the association with ischaemic stroke persists. This is consistent with the results of a prospective cohort study among Korean men. ${ }^{14}$ It suggests that the correlation between FBG and stroke is mainly contributed by an ischaemic stroke. A large-scale prospective study in China (including 96110 participants) indicated that both hyperglycaemic and hypoglycaemic are associated with an increased risk of incident intracerebral haemorrhage, after adjustment for potential confounders. ${ }^{24}$

Results based on the ADA and WHO criteria demonstrated that FBG levels of $\geq 7.0 \mathrm{mmol} / \mathrm{L}$ were a significant risk factor for stroke (HR $(95 \% \mathrm{CI}) 1.68$ (1.24 to 2.27) and 1.62 (1.21 to 2.13) following the ADA and WHO criteria, respectively), whereas the risk of stroke in patients with IFG had an increasing trend, but this trend was not statistically significant. A large number of studies showed that people with diabetes are at increased 


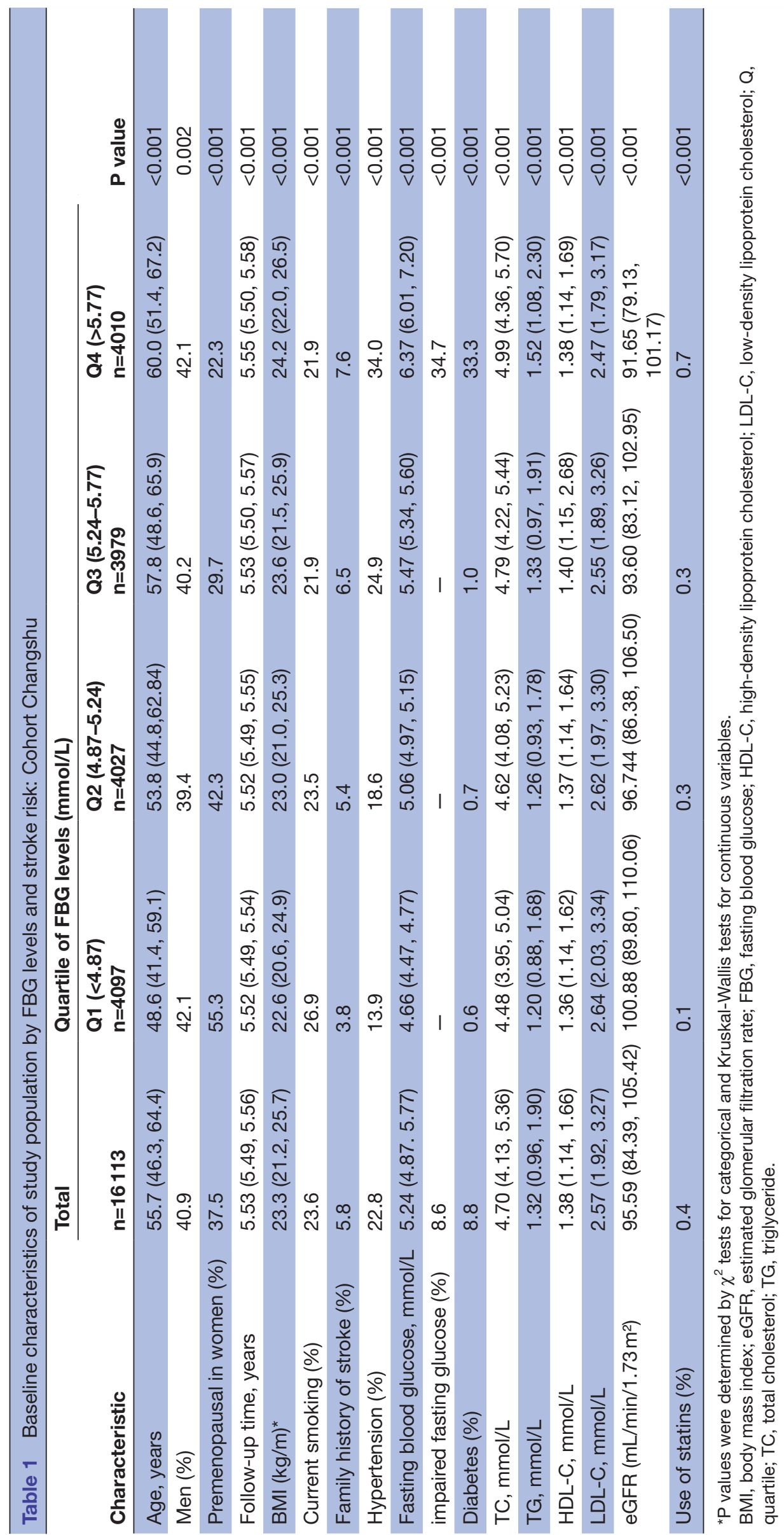


Table 2 HRs (95\% Cl) for incident stroke by FBG quartiles

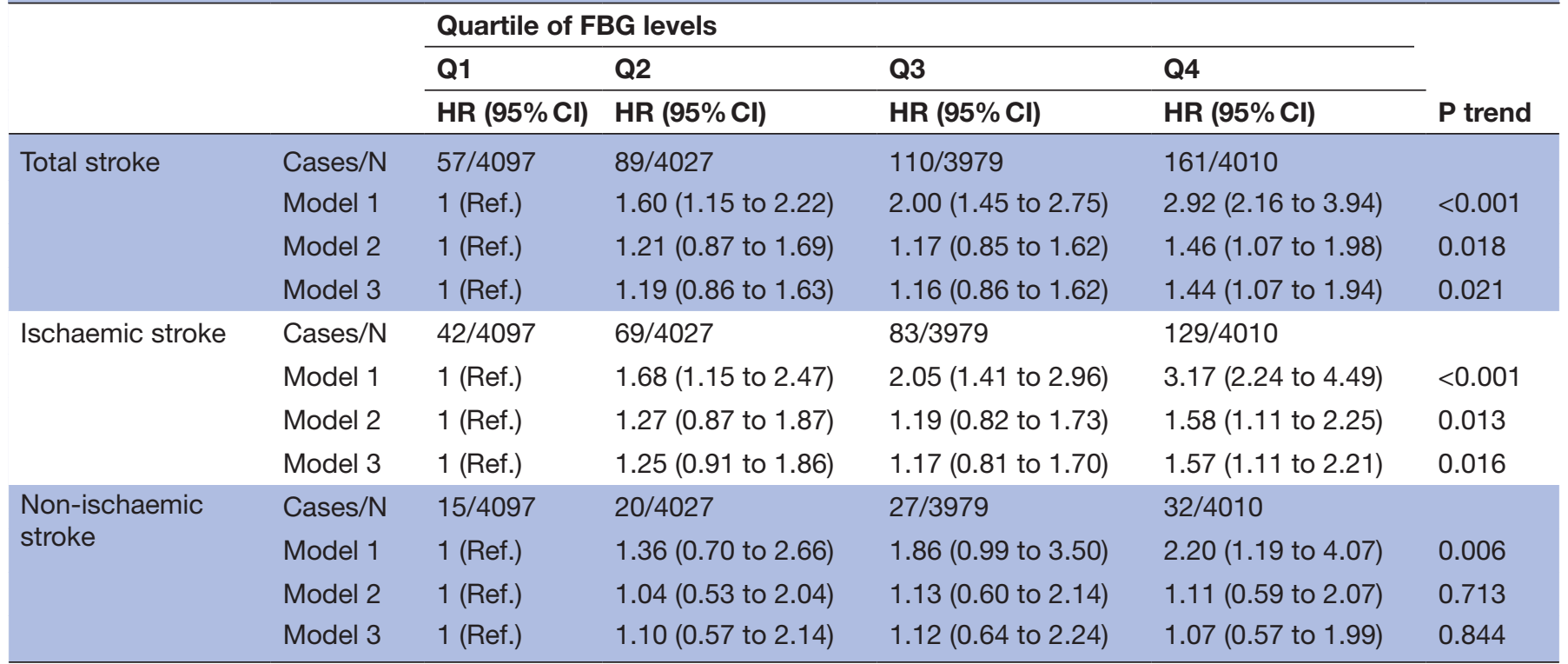

Model 1: unadjusted model; model 2: adjusted for age, sex and BMI; model 3: further adjusted for HDL-C, TC, TG, LDL-C, eGFR, hypertension, smoking status, use of statins and family history of stroke. eGFR, estimated glomerular filtration rate; FBG, fasting blood glucose; HDL-C, high-density lipoprotein cholesterol; LDL-C, low-density lipoprotein cholesterol; TC, total cholesterol; TG, triglyceride.

risk of ischaemic stroke. ${ }^{25}{ }^{26} \mathrm{~A}$ meta-analysis including 698782 participants reported that the risk of ischaemic stroke in diabetic patients was 2.27 times higher than normal people. ${ }^{25}$ A cohort study in Swedish also showed that poor blood glucose control in patients with type 2 diabetes was associated with an increased risk of stroke and death. ${ }^{26}$ Although diabetes is associated with stroke, the influence of pre-diabetes on future stroke risk has not been clear yet. Some large sample meta-analyses reported pre-diabetes is associated with the risk of $\mathrm{CVD}^{27} 28$ and heart failure. ${ }^{29}$ But in another meta-analysis, including 15 cohort studies, more than half of the studies showed that after adjusting for cardiovascular risk factors, there was no significant association between pre-diabetes and stroke. ${ }^{30}$

\begin{tabular}{|c|c|c|c|c|c|}
\hline & & & Model 1 & Model 2 & Model 3 \\
\hline \multirow[t]{7}{*}{ ADA definition } & $<5.6 \mathrm{mmol} / \mathrm{L}$ & Cases/N & $220 / 11079$ & & \\
\hline & & $\mathrm{HR}(95 \% \mathrm{Cl})$ & 1 (Ref.) & 1 (Ref.) & 1 (Ref.) \\
\hline & $5.6-6.9 \mathrm{mmol} / \mathrm{L}$ & Cases/N & $136 / 3869$ & & \\
\hline & & HR $(95 \% \mathrm{Cl})$ & 1.78 (1.43 to 2.20$)$ & $1.16(0.94$ to 1.44$)$ & 1.17 (0.93 to 1.42$)$ \\
\hline & $\geq 7.0 \mathrm{mmol} / \mathrm{L}$ & Cases/N & $61 / 1165$ & & \\
\hline & & HR $(95 \% \mathrm{Cl})$ & 2.69 (2.02 to 3.57$)$ & 1.72 (1.29 to 2.28$)$ & 1.68 (1.24 to 2.27$)$ \\
\hline & $\mathrm{P}$ trend & & $<0.001$ & 0.001 & 0.001 \\
\hline \multirow[t]{7}{*}{ WHO definition } & $<6.1 \mathrm{mmol} / \mathrm{L}$ & Cases/N & $301 / 13422$ & & \\
\hline & & $\mathrm{HR}(95 \% \mathrm{Cl})$ & 1 (Ref.) & 1 (Ref.) & 1 (Ref.) \\
\hline & $6.1-6.9 \mathrm{mmol} / \mathrm{L}$ & Cases/N & $55 / 1526$ & & \\
\hline & & $\mathrm{HR}(95 \% \mathrm{Cl})$ & 1.61 (1.21 to 2.15$)$ & $1.08(0.81$ to 1.45$)$ & 1.11 (0.83 to 1.47$)$ \\
\hline & $\geq 7.0 \mathrm{mmol} / \mathrm{L}$ & Cases/N & $61 / 1165$ & & \\
\hline & & $\mathrm{HR}(95 \% \mathrm{Cl})$ & 2.38 (1.81 to 3.03 ) & 1.64 (1.24 to 2.17$)$ & 1.62 (1.21 to 2.13 ) \\
\hline & $\mathrm{P}$ trend & & $<0.001$ & 0.001 & 0.002 \\
\hline
\end{tabular}

Model 1: unadjusted model; model 2: adjusted for age, sex and BMI; model 3: further adjusted for HDL-C, TC, TG, LDL-C, eGFR, hypertension, smoking status, use of statins and family history of stroke.

ADA, American Diabetes Association; eGFR, estimated glomerular filtration rate; FBG, fasting blood glucose; HDL-C, high-density lipoprotein cholesterol; LDL-C, low-density lipoprotein cholesterol; TC, total cholesterol; TG, triglyceride. 
Table 4 HRs $(95 \% \mathrm{Cl})$ for incident stroke by sex

\begin{tabular}{|c|c|c|c|c|c|c|}
\hline & & \multicolumn{4}{|c|}{ Quartile of FBG levels } & \multirow[b]{3}{*}{$\mathrm{P}$ trend } \\
\hline & & Q1 & Q2 & Q3 & Q4 & \\
\hline & & HR (95\% Cl) & HR $(95 \% \mathrm{Cl})$ & HR $(95 \% \mathrm{Cl})$ & HR $(95 \% \mathrm{Cl})$ & \\
\hline \multirow{2}{*}{ Men } & Model 1 & 1 (Ref.) & $1.40(0.89$ to 2.20$)$ & 1.74 (1.13 to 2.69$)$ & 1.99 (1.31 to 3.03$)$ & 0.001 \\
\hline & Model 2 & 1 (Ref.) & 1.04 (0.66 to 1.64$)$ & 1.16 (0.75 to 1.80$)$ & $1.11(0.73$ to 1.71$)$ & 0.560 \\
\hline \multirow[t]{4}{*}{ Women } & Cases/N & $24 / 2372$ & $47 / 2441$ & $57 / 2380$ & $97 / 2322$ & \\
\hline & Model 1 & 1 (Ref.) & 1.91 (1.21 to 3.29$)$ & 2.38 (1.48 to 3.84$)$ & 4.19 (2.68 to 6.55$)$ & $<0.001$ \\
\hline & Model 2 & 1 (Ref.) & $1.45(0.89$ to 2.37$)$ & 1.25 (0.78 to 2.03$)$ & $1.90(1.21$ to 2.99$)$ & 0.006 \\
\hline & Model 3 & 1 (Ref.) & 1.43 (0.87 to 2.33$)$ & 1.30 (0.80 to 2.09$)$ & $1.92(1.22$ to 3.01$)$ & 0.004 \\
\hline
\end{tabular}

Model 1: unadjusted model; model 2: adjusted for age, sex and BMI; model 3: further adjusted for HDL-C, TC, TG, LDL-C, eGFR, hypertension, smoking status, use of statins and family history of stroke.

BMI, body mass index; eGFR, estimated glomerular filtration rate; FBG, fasting blood glucose; HDL-C, high-density lipoprotein cholesterol; LDL-C, low-density lipoprotein cholesterol; TC, total cholesterol; TG, triglyceride.

A cohort study investigated the sex-specific associations of pre-diabetes with major clinical outcomes reached the same conclusion. ${ }^{31}$

Stratified analysis showed that the correlation between FBG levels and stroke risk only existed in women. According to the restratification of menopause, only postmenopausal women with the highest FBG level had a higher risk of stroke (HR (95\% CI) 1.68 (1.06 to 2.68)), no associations were found in premenopausal women. It is probably due to the low number of stroke events in premenopausal women. Few published studies reported sex-stratified results. A sex-specific cohort study in the Middle East showed that only men with higher FBG levels showed an increased risk of stroke (HR: 2.15 (1.26 to 4.67) in FBG level of 6.1-6.9 mmol/L, HR: 2.38 (1.08 to $5.25)$ in FBG level of $>7.0 \mathrm{mmol} / \mathrm{L}){ }^{32}$ Korean Heart Study

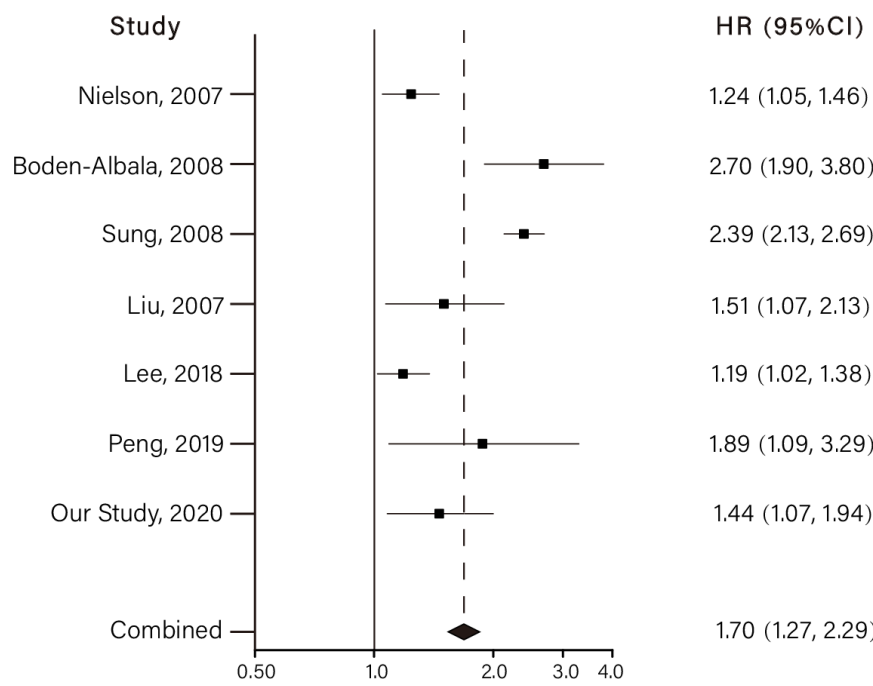

Figure 1 Meta-analysis of fasting blood glucose at the highest vs lowest levels and the risk of developing stroke. The horizontal lines indicate the lower and upper limits of the $95 \% \mathrm{Cl}$, and the grey squares reflect the HR of each study. showed that IFG was associated with an increased risk of ischaemic stroke in men but not in women. ${ }^{33}$ We suspect that the disparities in sex between studies could be due to different definitions of outcome (all stroke vs ischaemic stroke) and the variety in covariate adjustments. A metaanalysis including 64 cohort studies provides the clearest evidence yet for the sex difference of diabetes and stroke risk. The excess risk of diabetes-related stroke in women is significantly higher than that in men and independent of sex difference in other cardiovascular risk factors. ${ }^{34}$ Our results support the findings of this meta-analysis, indicating that the association between FBG levels and stroke cases in women, and the mechanism may be related to the protective effect of oestrogen.

\section{Possible mechanisms}

Accumulating evidence suggests that FBG levels can be used to predict the risk of CVD. ${ }^{202535}$ As an adverse consequence of CVD, stroke is closely related to FBG levels. ${ }^{3637}$ Its mechanism may be related to the dysfunction of cerebral microvasculature caused by hyperglycaemic, insulin resistance, obesity and hypertension. ${ }^{38}$ The microvasculature is involved in the regulation of many brain processes. Once damaged, it is easy to cause cerebrovascular accidents. ${ }^{39}$ The dysfunction is also obvious in the pre-diabetes population, which may suggest that IFG is the risk factor for stroke. ${ }^{40}$ Besides, diabetic retinopathy was proved to be a sign of lacunar ischaemic stroke, which had the same effects as cerebral microvascular dysfunction. ${ }^{41}$

Compared with men, women with diabetes are at higher risk of stroke. ${ }^{34}$ Previously, it was thought that the sex specificity of the severity of diabetes-related CVDs was caused by the differences between men and women in treatment and chronic disease management. However, as the gradual equality of men and women, the risk of serious CVD in postmenopausal women is still higher. ${ }^{34}$ Sex hormones may explain the sex differences observed 
in human epidemiological studies, ${ }^{42-44}$ indicating the oestrogen protection in women, preventing the progression of non-diabetic CVDs at least before menopause. Animal experiments show that the decrease in oestrogen levels in ovariectomised mice can lead to impaired glycaemia, reduced glucose tolerance, hyperinsulinemia and impaired insulin secretion, and then develop into hyperglycaemic and atherosclerosis. ${ }^{45}$ However, diabetes seemingly attenuates the protective effect of oestrogen on women in the development of CVD. ${ }^{46}$

\section{Strengths and limitations}

The main strengths of this study include the large sample size, measurement of plasma FBG and the complete follow-up. We followed up the cases by recording linkages with hospital discharge diagnoses and the Cause of Death Registry. We used both quartiles and clinical classifications (normal FBG, IFG, hyperglycaemic) to classify FBG levels and obtained similar results. We considered different stroke subtypes as the study outcome. To our knowledge, this study provides the first meta-analysis investigating the association between FBG levels and stroke risk in the general population; therefore, results are likely generalizable to all races.

Our study has several limitations. First, we do not have HbAlc measurements in this study. Among indicators to assess glucose variability, $\mathrm{HbAlc}$ is the gold standard for clinical evaluation of long-term blood glucose control, ${ }^{8}$ while FBG may overlook the potential effects of postprandial blood glucose levels and other meaningful factors. ${ }^{47}$ Reports revealed that HbA1c played an important role in distinguishing people with high cardiovascular risk from those without diabetes. ${ }^{48}{ }^{49}$ Second, the levels of estradiol and testosterone in the study subjects were not measured, although we speculated that the sex difference was probably due to the protective effect of sex hormones. Third, due to a low number of stroke cases, the real risk association of the premenopausal population may be biased.

\section{CONCLUSIONS}

Our study indicates that higher FBG levels are associated with an increased stroke in the general population and meta-analysis corroborated this finding. Furthermore, we observed that the association was more prominent in women than in men. We also observed strong risk associations among postmenopausal or oophorectomy women. FBG levels as a routine and low-cost measurement could be used to identify individuals with higher stroke risk in the future.

Contributors Conceptualisation: $\mathrm{YZ}$ and $\mathrm{HZ}$; methodology: $\mathrm{YZ}$; software, $\mathrm{YZ}$; validation: YZ; formal analysis: YZ; resources: $\mathrm{SG}, \mathrm{QZ}, \mathrm{MY}$ and ZZ; data curation: YZ; visualization: $Y Z$; investigation: $Y Z, C W$ and $D L$; writing —original draft preparation: $\mathrm{YZ}$ and SG; writing — review and editing: $\mathrm{YZ}$ and $\mathrm{HZ}$; supervision: $\mathrm{HZ}$; project administration: $\mathrm{HZ}$; funding acquisition: $\mathrm{SG}$ and $\mathrm{HZ}$. All authors have agreed on the journal to which the article has been submitted and agreed to be accountable for all aspects of the work. All authors read and approved the final manuscript.

Funding This work was supported by the National Natural Science Foundation of China (number 81973122).
Competing interests None declared.

Patient consent for publication Not required.

Ethics approval The study protocol conforms to the ethical guidelines of the Declaration of Helsinki. The baseline survey and record linkages with study outcomes were approved by the Ethics Committee of Fuwai Cardiovascular Hospital, Beijing, China (number 2012-399). After a detailed explanation of our study, we obtained a written informed consent form from each participant.

Provenance and peer review Not commissioned; externally peer reviewed.

Data availability statement Data may be obtained from a third party and are not publicly available. The datasets generated and/or analyzed during the current study are not publicly available due to the restrictions of containing information that could compromise the privacy of research participants.

Supplemental material This content has been supplied by the author(s). It has not been vetted by BMJ Publishing Group Limited (BMJ) and may not have been peer-reviewed. Any opinions or recommendations discussed are solely those of the author(s) and are not endorsed by BMJ. BMJ disclaims all liability and responsibility arising from any reliance placed on the content. Where the content includes any translated material, BMJ does not warrant the accuracy and reliability of the translations (including but not limited to local regulations, clinical guidelines, terminology, drug names and drug dosages), and is not responsible for any error and/or omissions arising from translation and adaptation or otherwise.

Open access This is an open access article distributed in accordance with the Creative Commons Attribution Non Commercial (CC BY-NC 4.0) license, which permits others to distribute, remix, adapt, build upon this work non-commercially, and license their derivative works on different terms, provided the original work is properly cited, appropriate credit is given, any changes made indicated, and the use is non-commercial. See: http://creativecommons.org/licenses/by-nc/4.0/.

ORCID iD

Ya Zhang http://orcid.org/0000-0002-9717-911X

\section{REFERENCES}

1 Feigin VL, Roth GA, Naghavi M, et al. Global burden of stroke and risk factors in 188 countries, during 1990-2013: a systematic analysis for the global burden of disease study 2013. Lancet Neurol 2016;15:913-24.

2 Feigin VL, Norrving B, Mensah GA. Global burden of stroke. Circ Res 2017;120:439-48.

3 Feigin VL, Krishnamurthi RV, Parmar P, et al. Update on the global burden of ischemic and hemorrhagic stroke in 1990-2013: the GBD 2013 study. Neuroepidemiology 2015;45:161-76.

4 Wang W, Jiang B, Sun H, et al. Prevalence, incidence, and mortality of stroke in china: results from a nationwide population-based survey of 480687 adults. Circulation 2017;135:759-71.

5 Liu L-P, Wong LKS, Wang DZ, et al. Current status of endovascular procedures in management of ischemic stroke in China. CNS Neurosci Ther 2014;20:483-4.

6 Banerjee C, Moon YP, Paik MC, et al. Duration of diabetes and risk of ischemic stroke: the Northern Manhattan study. Stroke 2012;43:1212-7.

7 Bragg F, Li L, Yang L, et al. Risks and population burden of cardiovascular diseases associated with diabetes in China: a prospective study of 0.5 million adults. PLoS Med 2016;13:e1002026.

8 Jia Z, Wu S, Zhou Y, et al. Metabolic syndrome and its components as predictors of stroke in middle-aged and elderly Chinese people. Neurol Res 2011;33:453-9.

9 Moebus S, Göres L, Lösch C, et al. Impact of time since last caloric intake on blood glucose levels. Eur J Epidemiol 2011;26:719-28.

10 Morris H. Standards of medical care in diabetes-2014. Diabetes Care 2014;36:S11-66.

11 Friedewald WT, Levy RI, Fredrickson DS. Estimation of the concentration of low-density lipoprotein cholesterol in plasma, without use of the preparative ultracentrifuge. Clin Chem 1972;18:499-502.

12 Levey AS, Stevens LA, Schmid CH, et al. A new equation to estimate glomerular filtration rate. Ann Intern Med 2009;150:604-12.

13 Chalmers J, MacMahon S, Mancia G, et al. 1999 World health Organization-International Society of hypertension guidelines for the management of hypertension. guidelines Sub-committee of the world Health organization. Clin Exp Hypertens 1999;21:1009-60. doi:10.3109/10641969909061028 
14 World Health Organization. Use of glycated haemoglobin (HbA1c) in diagnosis of diabetes mellitus: abbreviated report of a WHO consultation: World Health Organization 2011.

15 American Diabetes Association. Standards of medical care in diabetes-2020 abridged for primary care providers. Clin Diabetes 2020;38:10-38.

16 Kok P, Seidell JC, Meinders AE. [The value and limitations of the body mass index (BMI) in the assessment of the health risks of overweight and obesity]. Ned Tijdschr Geneeskd 2004;148:2379-82.

17 Nielson C, Fleming RM. Blood glucose and cerebrovascular disease in nondiabetic patients. Angiology 2007;58:625-9.

18 Boden-Albala B, Cammack S, Chong J, et al. Diabetes, fasting glucose levels, and risk of ischemic stroke and vascular events: findings from the Northern Manhattan study (NOMAS). Diabetes Care 2008;31:1132-7.

19 Sung J, Song Y-M, Ebrahim S, et al. Fasting blood glucose and the risk of stroke and myocardial infarction. Circulation 2009;119:812-9.

20 Liu F, Yang X, Li J, et al. Association of fasting glucose levels with incident atherosclerotic cardiovascular disease: an 8-year follow-up study in a Chinese population. J Diabetes 2017;9:14-23.

21 Lee G, Kim SM, Choi S, et al. The effect of change in fasting glucose on the risk of myocardial infarction, stroke, and all-cause mortality: a nationwide cohort study. Cardiovasc Diabetol 2018;17:51.

22 Peng X, Ge J, Wang C, et al. Longitudinal average glucose levels and variance and risk of stroke: a Chinese cohort study. Int J Hypertens 2020;2020:1-8.

23 Begg CB, Mazumdar M. Operating characteristics of a RANK correlation test for publication bias. Biometrics 1994;50:1088-101.

24 Jin C, Li G, Rexrode KM, et al. Prospective study of fasting blood glucose and intracerebral hemorrhagic risk. Stroke 2018;49:27-33.

25 Emerging Risk Factors Collaboration, Sarwar N, Gao P, et al. Diabetes mellitus, fasting blood glucose concentration, and risk of vascular disease: a collaborative meta-analysis of 102 prospective studies. Lancet 2010;375:2215-22.

26 Zabala A, Darsalia V, Holzmann MJ, et al. Risk of first stroke in people with type 2 diabetes and its relation to glycaemic control: a nationwide observational study. Diabetes Obes Metab 2020;22:182-90.

27 Cai X, Zhang Y, Li M, et al. Association between prediabetes and risk of all cause mortality and cardiovascular disease: updated metaanalysis. BMJ 2020;370:m2297

28 Huang Y, Cai X, Mai W, et al. Association between prediabetes and risk of cardiovascular disease and all cause mortality: systematic review and meta-analysis. BMJ 2016;355:i5953.

29 Cai X, Liu X, Sun L, et al. Prediabetes and the risk of heart failure: a meta-analysis. Diabetes Obes Metab 2021;23:1746-53.

30 Lee M, Saver JL, Hong K-S, et al. Effect of pre-diabetes on future risk of stroke: meta-analysis. BMJ 2012;344:e3564.

31 Shang Y, Fratiglioni L, Marseglia A, et al. Association of diabetes with stroke and post-stroke dementia: a population-based cohort study. Alzheimers Dement 2020;16:1003-12.

32 Parizadeh D, Rahimian N, Akbarpour S, et al. Sex-Specific clinical outcomes of impaired glucose status: a long follow-up from the Tehran lipid and glucose study. Eur J Prev Cardiol 2019;26:1080-91.
$33 \mathrm{Kim} \mathrm{H-K}, \mathrm{Kim} \mathrm{C}-\mathrm{H}$, Kim EH, et al. Impaired fasting glucose and risk of cardiovascular disease in Korean men and women: the Korean heart study. Diabetes Care 2013;36:328-35.

34 Peters SAE, Huxley RR, Woodward M. Diabetes as a risk factor for stroke in women compared with men: a systematic review and metaanalysis of 64 cohorts, including 775,385 individuals and 12,539 strokes. Lancet 2014;383:1973-80.

35 Bragg F, Li L, Smith M, et al. Associations of blood glucose and prevalent diabetes with risk of cardiovascular disease in 500 000 adult Chinese: the China Kadoorie Biobank. Diabet Med 2014;31:540-51.

36 Luitse MJA, Biessels GJ, Rutten GEHM, et al. Diabetes, hyperglycaemia, and acute ischaemic stroke. Lancet Neurol 2012;11:261-71.

37 Liu J, Rutten-Jacobs L, Liu M, et al. Causal impact of type 2 diabetes mellitus on cerebral small vessel disease: a Mendelian randomization analysis. Stroke 2018;49:1325-31.

38 van Sloten TT, Sedaghat S, Carnethon MR, et al. Cerebral microvascular complications of type 2 diabetes: stroke, cognitive dysfunction, and depression. Lancet Diabetes Endocrinol 2020;8:325-36

39 Wardlaw JM, Smith C, Dichgans M. Small vessel disease: mechanisms and clinical implications. Lancet Neurol 2019;18:684-96.

40 Stehouwer CDA, Dysfunction M. Microvascular dysfunction and hyperglycemia: a vicious cycle with widespread consequences. Diabetes 2018;67:1729-41.

41 Hankey GJ, Anderson NE, Ting R-D, et al. Rates and predictors of risk of stroke and its subtypes in diabetes: a prospective observational study. J Neurol Neurosurg Psychiatry 2013;84:281-7.

42 Brann DW, Dhandapani K, Wakade C, et al. Neurotrophic and neuroprotective actions of estrogen: basic mechanisms and clinical implications. Steroids 2007;72:381-405.

43 Howard VJ, Madsen TE, Kleindorfer DO, et al. Sex and race differences in the association of incident ischemic stroke with risk factors. JAMA Neurol 2019;76:179-86.

44 Reeves MJ, Bushnell CD, Howard G, et al. Sex differences in stroke: epidemiology, clinical presentation, medical care, and outcomes. Lancet Neurol 2008;7:915-26.

45 Venegas-Pino DE, Wang P-W, Stoute HK, et al. Sex-Specific Differences in an ApoE(-/-):Ins2(+/Akita) Mouse Model of Accelerated Atherosclerosis. Am J Pathol 2016;186:67-77.

46 Kautzky-Willer A, Harreiter J, Pacini G. Sex and gender differences in risk, pathophysiology and complications of type 2 diabetes mellitus. Endocr Rev 2016;37:278-316.

47 Jia $\mathrm{Q}$, Zheng $\mathrm{H}$, Zhao X, et al. Abnormal glucose regulation in patients with acute stroke across China: prevalence and baseline patient characteristics. Stroke 2012;43:650-7.

48 Di Pino A, Mangiafico S, Urbano F, et al. Hba1C identifies subjects with prediabetes and subclinical left ventricular diastolic dysfunction J Clin Endocrinol Metab 2017;102:3756-64.

49 Selvin E, Steffes MW, Zhu H, et al. Glycated hemoglobin, diabetes, and cardiovascular risk in nondiabetic adults. $N$ Engl J Med 2010;362:800-11. 